\title{
The Infimum Norm of Completely Positive Maps
}

\author{
Ching Yun Suen
}

Correspondence: Ching-Yun Suen, Foundational Sciences, Texas A\&M University at Galveston, P. O. Box 1675, Galveston, Texas 77553-1675, USA.

Received: October 17, 2021 Accepted: December 3, 2021 Online Published: January 14, 2022

doi:10.5539/jmr.v13n6p51

URL: https://doi.org/10.5539/jmr.v13n6p51

\section{Abstract}

Let $A$ be a unital $C^{*}$-algebra, let $L: A \rightarrow B(H)$ be a linear map, and let $\emptyset: A \rightarrow B(H)$ be a completely positive linear map. We prove the property in the following: $\inf \left\{\|\varnothing\|:\left(\begin{array}{ccc}\varnothing & L & 0 \\ L^{*} & \varnothing & L \\ 0 & L^{*} & \varnothing\end{array}\right)\right.$ is completely positive $\}=\inf \left\{\| T^{*} T+\right.$ $T T^{*} \|^{\frac{1}{2}}: L=V^{*} T \pi V$ which is a minimal commutant representation with isometry $\}$. Moreover, if $L=L^{*}$, then $\inf \left\{\|\varnothing\|:\left(\begin{array}{ccc}\varnothing & L & 0 \\ L & \varnothing & L \\ 0 & L & \varnothing\end{array}\right)\right.$ is completely positive $\}=\sqrt{2}\|L\|_{c b}$. In the paper we also extend the result $\inf \left\{\|\varnothing\|:\left(\begin{array}{cc}\emptyset & L \\ L^{*} & \emptyset\end{array}\right)\right.$ is completely positive $\}=\inf \left\{\|T\|: L=V^{*} T \pi V\right\}$ [3, Corollary 3.12].

Keywords: positive operators, completely positive maps, completely bounded maps

AMS Subject Classification (2010): 47A63

\section{Introduction}

Let $M_{n}$ denote the $C^{*}$-algebra of complex $n \times n$ matrices and $B(H)$ the algebra of all bounded linear operators on a Hilbert space $H$.Let $A$ and $B$ be $C^{*}$-algebras and let $L: A \rightarrow B$ be a bounded map linear map. The map $L$ is called positive if $L(a)$ is positive whenever $a$ is positive. The map $L$ is called completely positive if $L \otimes I_{n}: A \otimes M_{n} \rightarrow$ $B \otimes M_{n}$ defined by $L \otimes I_{n}(a \otimes b)=L(a) \otimes b$ is positive for all $n$. From [3], $\|L\|_{w_{\rho}}=\sup \left\{w_{\rho}(L(a)): w_{\rho}(a) \leq\right.$ 1 ). The map $L$ is $w_{\rho}$ completely bounded if $\sup _{n}\left\|L \otimes I_{n}\right\|_{w_{\rho}}$ is finite. Notice that $\|L\|_{c b}=\|L\|_{w_{1} c b}$ and $\|L\|_{w c b}=$ $\|L\|_{w_{2} c b}$. The map $L=L^{*}$ if $L(a)=L\left(a^{*}\right)^{*}$. From [2], we know that every completely bounded map $L$ from $A$ to $B(H)$ has a minimal commutant representation $L=V^{*} T \pi V($ m.c.r. $i)$ with $T$ in the commutant of $\pi(A)$ and isometry $V$. In the paper we obtain a lower bound for the set $\left\{\|\varnothing\|:\left(\begin{array}{cccc}\varnothing & L & 0 & 0 \\ L^{*} & \emptyset & \ddots & 0 \\ 0 & \ddots & \ddots & L \\ 0 & 0 & L^{*} & \varnothing\end{array}\right)\right.$ is completely positive with $\left.m \geq 2\right\}$ which extends the property [3] $\inf \left\{\|\emptyset\|:\left(\begin{array}{cc}\varnothing & L \\ L^{*} & \emptyset\end{array}\right)\right.$ is completely positive $\}=\inf \left\{\|T\|: L=V^{*} T \pi V(m . c . r . i)\right\}$. In particular, we have the value of $\inf \left\{\|\varnothing\|:\left(\begin{array}{ccc}\emptyset & L & 0 \\ L^{*} & \varnothing & L \\ 0 & L^{*} & \varnothing\end{array}\right)\right.$ is completely positive $\}$ in the paper.

\section{Infimum Norm}

Proposition 2.1. Let $A$ be a unital $C^{*}$-algebra and $L: A \rightarrow B(H)$ be a completely bounded map, then inf $\{\|\varnothing\|$ : 
$\left(\begin{array}{cccc}\emptyset & L & 0 & 0 \\ L^{*} & \emptyset & \ddots & 0 \\ 0 & \ddots & \ddots & L \\ 0 & 0 & L^{*} & \emptyset\end{array}\right)_{m \times m} \quad$ is completely positive $\}=\inf \left\{k:\left(\begin{array}{cccc}k & T & 0 & 0 \\ T^{*} & k & \ddots & 0 \\ 0 & \ddots & \ddots & T \\ 0 & 0 & T^{*} & k\end{array}\right)_{m \times m}\right.$ is positive, $L=V^{*} T \pi V$ (m.c.r.i)\}, $m \geq 2$. Proof. If $\left(\begin{array}{cccc}\varnothing & L & 0 & 0 \\ L^{*} & \emptyset & \ddots & 0 \\ 0 & \ddots & \ddots & L \\ 0 & 0 & L^{*} & \emptyset\end{array}\right)_{m \times m}$ is completely positive, from the proof of [4, Theorem 2.6], the matrix $\left(\begin{array}{cccc}\|\varnothing\| & T & 0 & 0 \\ T & \|\varnothing\| & \ddots & 0 \\ 0 & \ddots & \ddots & T \\ 0 & 0 & T^{*} & \|\varnothing\|\end{array}\right)_{m \times m}$ is positive, where $L=V^{*} T \pi V$ (m.c.r.i) with an isometry $V$, a *-representation $\pi$, and $T$ in the commutant of $\pi(A)$. Conversely, if $\left(\begin{array}{cccc}k & T & 0 & 0 \\ T^{*} & k & \ddots & 0 \\ 0 & \ddots & \ddots & T \\ 0 & 0 & T^{*} & k\end{array}\right)_{m \times m}$ is positive and $L=V^{*} T \pi V$ (m.c.r.i), by [2, Proposition 2.6], we have $\left(\begin{array}{ccccc}k V^{*} \pi V & L & 0 & 0 \\ L^{*} & k V^{*} \pi V & & \ddots & 0 \\ 0 & \ddots & \ddots & L \\ 0 & 0 & L^{*} & k V^{*} \pi V\end{array}\right)_{m \times m}$ is completely positive, where $V^{*} \pi V$ is a unital completely positive.

Corollary 2.2. [3] $\inf \left\{\|\emptyset\|:\left(\begin{array}{cc}\varnothing & L \\ L^{*} & \emptyset\end{array}\right)\right.$ is completely positive $\}=\inf \left\{\|T\|: L=V^{*} T \pi V\right.$ (m.c.r.i) $\}=\|L\|_{w_{2} c b}$.

Proof. Let $m=2$ in Proposition 2.1.

Lemma 2.3. $\left(\begin{array}{ccc}k & T & 0 \\ T^{*} & k & T \\ 0 & T^{*} & k\end{array}\right) \geq 0$ if and only if $k^{2} I \geq T^{*} T+T T^{*}$ where $k>0$.

Proof. Since $\left(\begin{array}{cc}k & T \\ T^{*} & k\end{array}\right) \geq \frac{1}{k}(T, 0)^{*}(T, 0)$, we have $\left(\begin{array}{cc}k-\frac{1}{k} T^{*} T & T \\ T^{*} & k\end{array}\right) \geq 0$.

Since $\left(\begin{array}{cc}k & T^{*} \\ T & k-\frac{1}{k} T^{*} T\end{array}\right) \geq 0$, we have $k-\frac{1}{k} T^{*} T \geq \frac{1}{k} T T^{*}$.

Lemma 2.4. [5] $\min \left\{k:\left(\begin{array}{ccc}k & T & 0 \\ T^{*} & k & T \\ 0 & T^{*} & k\end{array}\right) \geq 0\right\}=\left\|T^{*} T+T T^{*}\right\|^{\frac{1}{2}}$.

Proof. If $\left(\begin{array}{ccc}k & T & 0 \\ T^{*} & k & T \\ 0 & T^{*} & k\end{array}\right) \geq 0$, applying Lemma 2.3 , we have $k \geq\left\|T^{*} T+T T^{*}\right\|^{\frac{1}{2}}$.

Since $\left(\left\|\mathrm{T}^{*} \mathrm{~T}+\mathrm{TT}^{*}\right\|^{\frac{1}{2}}\right)^{2} I \geq T^{*} T+T T^{*}$, applying Lemma 2.3, we have

$$
\left(\begin{array}{ccc}
\left\|\mathrm{T}^{*} \mathrm{~T}+\mathrm{TT}^{*}\right\|^{\frac{1}{2}} & T & 0 \\
T^{*} & \left\|\mathrm{~T}^{*} \mathrm{~T}+\mathrm{TT}^{*}\right\|^{\frac{1}{2}} & T \\
0 & T^{*} & \left\|\mathrm{~T}^{*} \mathrm{~T}+\mathrm{TT}^{*}\right\|^{\frac{1}{2}}
\end{array}\right) \geq 0 .
$$

Theorem 2.5. $\inf \left\{\|\emptyset\|:\left(\begin{array}{ccc}\emptyset & L & 0 \\ L^{*} & \emptyset & L \\ 0 & L^{*} & \emptyset\end{array}\right)\right.$ is completely positive $\}=\inf \left\{\left\|T^{*} T+T T^{*}\right\|^{\frac{1}{2}}: L=V^{*} T \pi V\right.$ (m.c.r.i) $\}$.

Proof. Let $m=3$ in Proposition 2.1, applying Lemma 2.4, we have the Theorem. 
Lemma 2.6. Let $T \in B(H)$. Then $2 w\left(S_{m}\right) S(L) \leq \inf \left\{\|\emptyset\|:\left(\begin{array}{cccc}\varnothing & L & 0 & 0 \\ L^{*} & \emptyset & \ddots & 0 \\ 0 & \ddots & \ddots & L \\ 0 & 0 & L^{*} & \emptyset\end{array}\right)_{m \times m}\right.$ is completely positive $\} \leq$ $2 w\left(S_{m}\right)\|L\|_{w_{2} c b} \quad(m \geq 2)$, where $S_{m}=\left(\begin{array}{cccc}0 & 1 & 0 & 0 \\ 0 & 0 & \ddots & 0 \\ \vdots & \ddots & \ddots & 1 \\ 0 & . & 0 & 0\end{array}\right)_{m \times m} \quad, S(L)=\inf \left\{w(T): L=V^{*} T \pi V(m . c . r . i)\right\}$, and $[3$, Corollary 3.12] $\|L\|_{w_{2} c b}=\inf \left\{\|T\|: L=V^{*} T \pi V\right.$ (m.c.r.i) $\}$.

Proof. From [6], we know that $2 w\left(S_{m}\right) w(T) \leq \inf \left\{k:\left(\begin{array}{cccc}k & T & 0 & 0 \\ T^{*} & k & \ddots & 0 \\ 0 & \ddots & \ddots & T \\ 0 & 0 & T^{*} & k\end{array}\right)_{m \times m}\right.$ is positive $\} \leq 2 w\left(S_{m}\right)\|T\|$ with $m \geq 2$. Applying Proposition 2.1, we have $2 w\left(S_{m}\right) \inf \left\{w(T): L=V^{*} T \pi V(m . c . r . i)\right\} \leq \inf \{\|\varnothing\|:$ $\left(\begin{array}{cccc}\emptyset & L & 0 & 0 \\ L^{*} & \emptyset & \ddots & 0 \\ 0 & \ddots & \ddots & L \\ 0 & 0 & L^{*} & \emptyset\end{array}\right)_{m \times m} \quad$ is completely positive $\} \leq 2 w\left(S_{m}\right) \inf \left\{\|T\|: L=V^{*} T \pi V\right.$ (m.c.r.i $\left.)\right\}$. Applying [4, Theorem 2.6] and [3, Corollary 3.12], we have the Lemma.

Theorem 2.7. If $L=L^{*}$, then inf $\left\{\|\varnothing\|:\left(\begin{array}{cccc}\varnothing & L & 0 & 0 \\ L & \varnothing & \ddots & 0 \\ 0 & \ddots & \ddots & L \\ 0 & 0 & L & \varnothing\end{array}\right)_{m \times m} \quad\right.$ is completely positive $\}=\inf \{k$ : $\left(\begin{array}{cccc}k & T & 0 & 0 \\ T & k & \ddots & 0 \\ 0 & \ddots & \ddots & T \\ 0 & 0 & T & k\end{array}\right)_{m \times m} \quad$ is positive and $\left.L=V^{*} T \pi V(m . c . r . i)\right\}=2 w\left(S_{m}\right)\|L\|_{c b}$.

Proof. Applying Proposition 2.1, Lemma 2.6, and [3, Corollary 3.3], we have the Theorem.

Corollary 2.8. If $L=L^{*}$, then $\inf \left\{\|\varnothing\|:\left(\begin{array}{ccc}\varnothing & L & 0 \\ L & \varnothing & L \\ 0 & L & \varnothing\end{array}\right)\right.$ is completely positive $\}=\sqrt{2}\|L\|_{c b}$.

Proof. From [1], $\quad w\left(S_{3}\right)=\cos \frac{\pi}{4}$.

Corollary 2.9. If $L=L^{*}$, then $\inf \left\{\|\emptyset\|:\left(\begin{array}{cccc}\varnothing & L & 0 & 0 \\ L & \emptyset & \ddots & 0 \\ 0 & \ddots & \ddots & L \\ 0 & 0 & L & \emptyset\end{array}\right)_{m \times m} \quad\right.$ is completely positive for all $\left.m \geq 2\right\}=2\|L\|_{c b}$.

Proof. $\lim _{m \rightarrow \infty} w\left(S_{m}\right)=\lim _{m \rightarrow \infty} \cos \frac{\pi}{m+1}$.

Example 2.10. Let $L: C \rightarrow M_{2}(C)$ be defined by $L(z)=\left(\begin{array}{ll}0 & z \\ Z & 0\end{array}\right)$ and $\emptyset: C \rightarrow M_{2}(C)$ be defined by $\emptyset(z)=\left(\begin{array}{ll}Z & 0 \\ 0 & Z\end{array}\right)$. Then $L$ is completely bounded and $\varnothing$ is a unital completely positive. Since $L^{*}=L$ and the map $\left(\begin{array}{ll}\varnothing & L \\ L & \varnothing\end{array}\right)$ is completely positive, by [3, Corollary 3.3 and Corollary 3.12], we have $\|L\|_{c b}=\|L\|_{w c b}=\|\varnothing\|=1$. Hence $\inf \left\{\|\varnothing\|:\left(\begin{array}{lll}\varnothing & L & 0 \\ L & \varnothing & L \\ 0 & L & \emptyset\end{array}\right)\right.$ is completely positive $\}=\sqrt{2}, \inf \left\{\|\varnothing\|:\left(\begin{array}{cccc}\emptyset & L & 0 & 0 \\ L & \emptyset & \ddots & 0 \\ 0 & \ddots & \ddots & L \\ 0 & 0 & L & \emptyset\end{array}\right)_{m \times m}\right.$ is completely positive with $m \geq 2\}=2 \cos \frac{\pi}{m+1}$, 
and $\inf \left\{\|\varnothing\|:\left(\begin{array}{cccc}\varnothing & L & 0 & 0 \\ L & \emptyset & \ddots & 0 \\ 0 & \ddots & \ddots & L \\ 0 & 0 & L & \varnothing\end{array}\right)_{m \times m}\right.$ is completely positive for all $\left.m \geq 2\right\}=2$.

\section{References}

Haagerup, U., \& de la Harpe, P. (1992). The numerical radius of a nilpotent operator on a Hilbert space. Proc. Amer. Math. Soc., 115, 371-379.

Paulsen, V. I., \& Suen, C. Y. (1985). Commutant representation of completely bounded maps. J. Operator Theory, 13, $87-101$.

Suen, C. Y. The minimum numbers for certain positive operators, to appear. Jour. Math. Res..

Suen, C. Y. (1995). The minimum norm of certain completely positive maps.Proc. Amer. Math Soc., 123, 2407-2416.

Suen, C. Y. (2000). Induced completely bounded norms and inflated Schur product. Acta Sci. Math. (Szeged), 66, 273-286.

Suen, C. Y. (2018). Strict positivity of operators and inflated Schur products. Jour. Math. Res., 10, 30-42.

\section{Copyrights}

Copyright for this article is retained by the author(s), with first publication rights granted to the journal.

This is an open-access article distributed under the terms and conditions of the Creative Commons Attribution license (http://creativecommons.org/licenses/by/4.0/). 